\title{
UAV-Based Sensor Web Monitoring System
}

\author{
Masahiko Nagai, ${ }^{1}$ Apichon Witayangkurn, ${ }^{2}$ Kiyoshi Honda, ${ }^{3}$ and Ryosuke Shibasaki ${ }^{2}$ \\ ${ }^{1}$ Asian Institute of Technology, Geoinformatics Center, P.O. Box 4, Klong Luang Pathumthani, 12120, Thailand \\ ${ }^{2}$ Center for Spatial Information Science, CSIS, The University of Tokyo, 5-1-5, Kashiwanoha, Kashiwa-shi, Chiba 277-8568, Japan \\ ${ }^{3}$ International Digital Earth Applied Science Research Center, Chubu University, 1200 Matsumoto-cho, Kasugai, Aichi 487-8501, Japan \\ Correspondence should be addressed to Masahiko Nagai, nagaim@ait.ac.th
}

Received 1 September 2011; Revised 20 December 2011; Accepted 17 January 2012

Academic Editor: Nicolas Sifakis

Copyright ( $) 2012$ Masahiko Nagai et al. This is an open access article distributed under the Creative Commons Attribution License, which permits unrestricted use, distribution, and reproduction in any medium, provided the original work is properly cited.

\begin{abstract}
An unmanned aerial vehicle- (UAV-) based monitoring system is developed as an intermediate system between aerial survey and ground survey. All the measurement tools are mounted on the UAV to acquire detailed information from low altitudes which is different from a satellite or a plane. The monitoring is carried out from the sky, but the spatial and temporal resolutions are freely selected near the ground. In this study, the data is easily acquired with safety and mobility by the utilization of a sensor web. A sensor web is a type of sensor network which is well suited for environmental monitoring. Sensor nodes are spatially distributed and wirelessly communicate with each other. In this study, the UAV-based system is considered as a mobile sensor node. This study proposes a combination of UAV-based monitoring with a ubiquitous sensor network.
\end{abstract}

\section{Introduction}

To obtain both the wide-area coverage of remote sensors and the high levels of detail and accuracy of ground surveying, at a low cost, an unmanned aerial vehicle- (UAV-) based monitoring system is developed. The UAV resembles a helicopter and all the measurement tools are mounted under it to acquire detailed information from low altitudes, unlike high-altitude systems in satellites or airplanes. The survey is conducted from the sky, but the resolution and accuracy are equal to those of ground surveying. Moreover, the UAV can acquire data easily as well as safely, for example, data from pollution monitoring, forest fire detection, and disaster monitoring [1]. On the other hand, a sensor web is a group or system of sensors, which connect together using a communication infrastructure to exchange information. It consists of multiple sensor stations which can be called sensor nodes. All sensor nodes can link or synchronize data among each other or the main station so that it acts as a network which is deployed in the area where monitoring is wanted and data, such as weather and environmental information, is collected. The main station is usually installed in rural areas or disaster sites [2].
Recently, much UAV research has been conducted with the existing UAV system which is designed to work as a standalone system [3]; thus making it difficult to be interoperable with other ground monitoring stations. This is where a sensor network can come into play. However, field sensor nodes currently in implementation by the sensor network support only fixed-position sensors that is not flexible enough for some field operations, such as adhoc node or mobile node [4].

In this study, a sensor web is applied for UAV-based monitoring. A UAV-based sensor web monitoring system is considered a mobile sensor node in a ubiquitous sensor network. This study proposes a combination of UAVbased monitoring with a ubiquitous sensor network for environment or disaster monitoring. In previous studies, wireless communication is used to transfer data to the base station for use by the pilot and the control center [1]. The aim of this study is to extend and publish local observations on the Internet and for it to be viewed by all concerned persons as a sensor node of a sensor network.

The main goal of this study is to design and develop a UAV system as a node of a field sensor network based on sensor observation service station (SOS Station) and sensor 


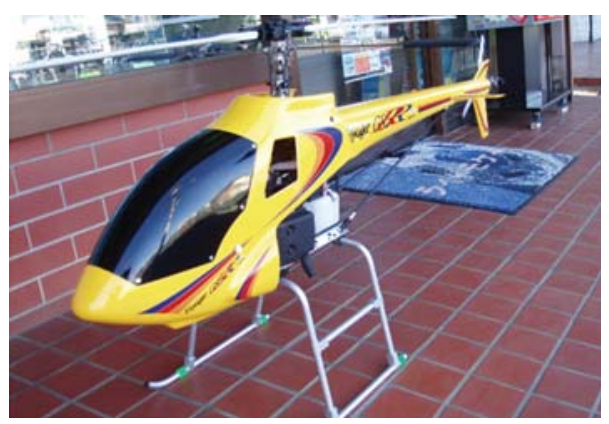

(a)

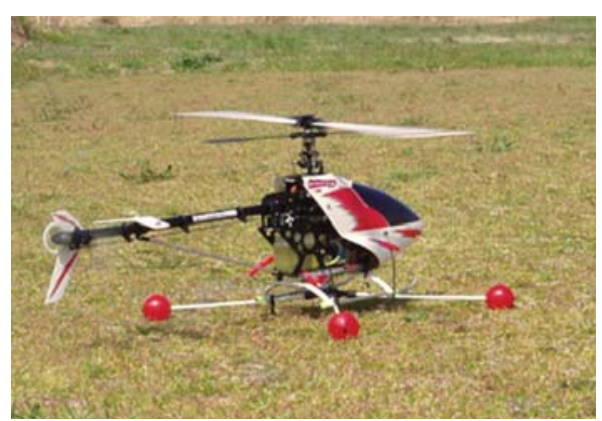

(b)

FIgURE 1: Voyager GSR 260 and Air Skipper 30, JR PROPO.

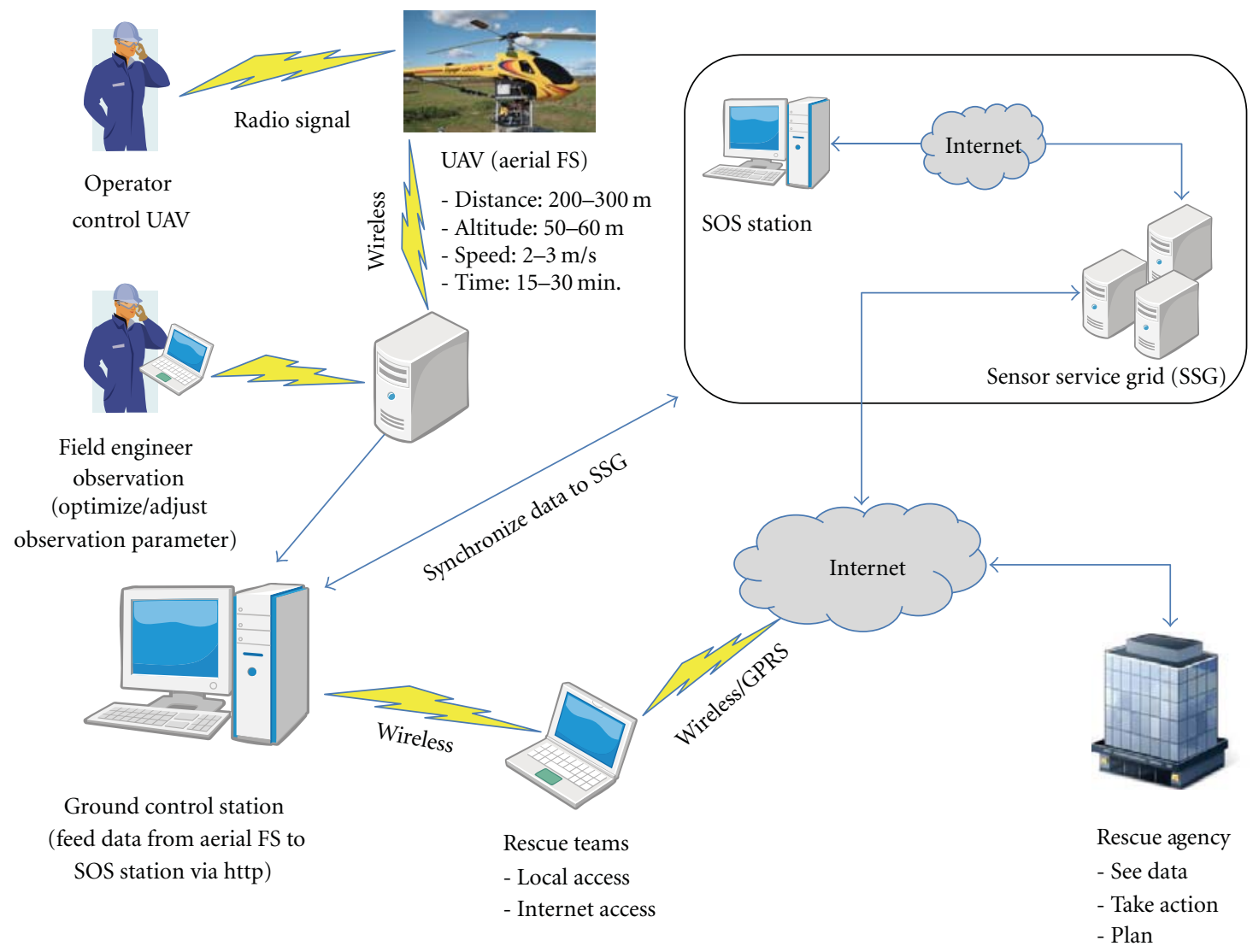

FIGURE 2: System architecture.

service grid (SSG). The aim is to achieve the following tasks: (1) design and implement a UAV system for realtime monitoring including framework, architecture and components; (2) connect sensors and geosensors such as GPS, gyroscope compass, and digital camera and laser range meter to a main controller for acquiring, processing and sending data to a ground station unit; (3) extend the UAV system to communicate with SOS and SSG so that it can act as one sensor node; (4) modify the sensor observation service station (SOS Station) and sensor service grid (SSG) to support mobile stations; (5) Test and evaluate the system in a flight experiment.

\section{Utilization of UAV and Sensor Web}

In this study, Voyager GSR 260 which is produced by JR PROPO, Japan, is used as a UAV platform. This is a $26 \mathrm{cc}$ gasoline engine helicopter, as shown in Figure 1. Overall length is $1,570 \mathrm{~mm}$, width is $386 \mathrm{~mm}$, and height is $670 \mathrm{~mm}$. Main rotor blade diameter is $1,770 \mathrm{~mm}$, and payload is $3-5 \mathrm{~kg}$. The price of this UAV is about US\$3,000. Air Skipper 30 is also used for this study to develop a more compact system. It is much smaller and cheaper than Voyager GSR 260 , costing about US\$1,500. There are several advantages to utilizing a UAV. One of the most important is that it is 


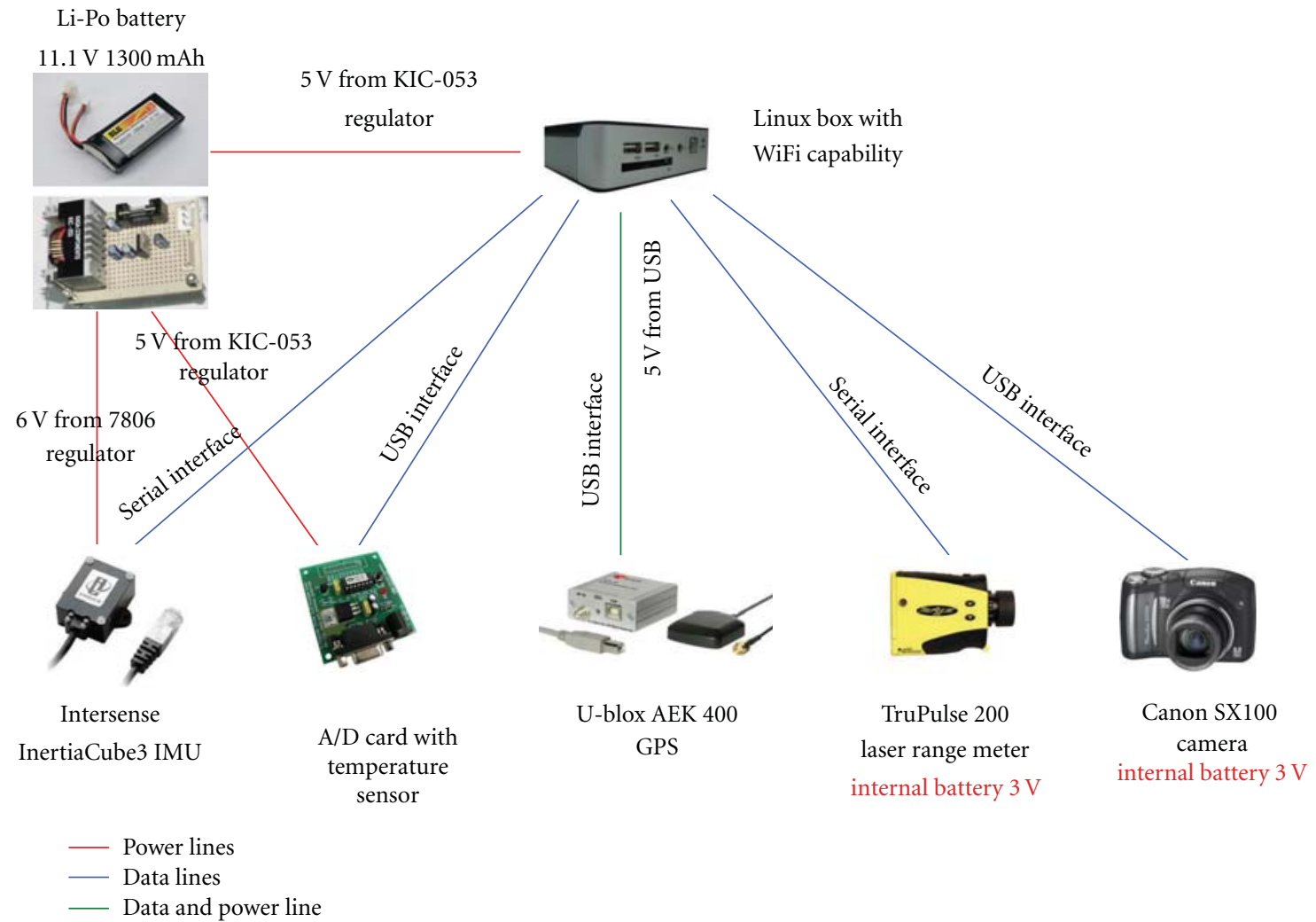

FIGURE 3: Summary of power connections and data lines.

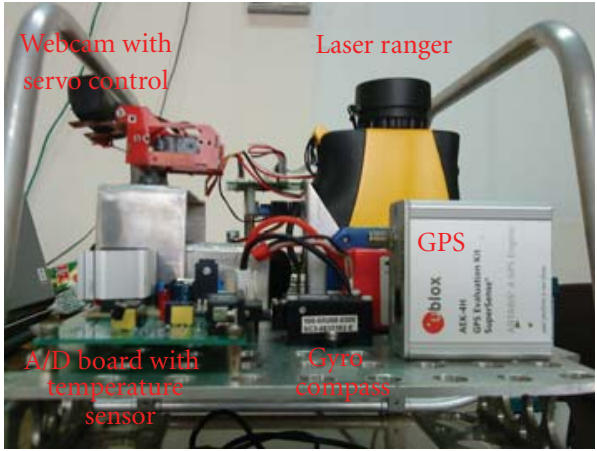

(a)

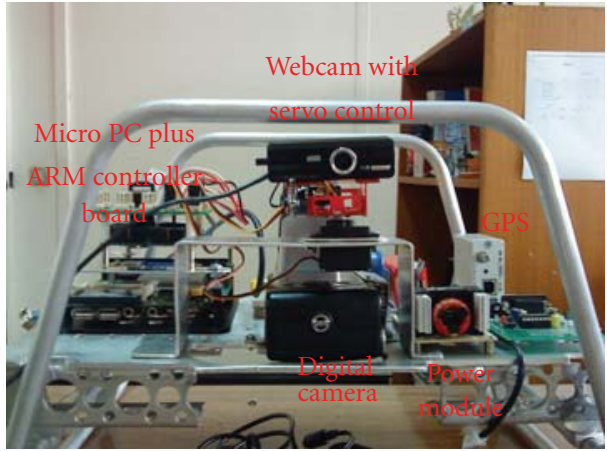

(b)

FIgURE 4: Sensor settings.

unmanned and, therefore, can fly over dangerous zones. This advantage suits a sensor web system as a sensor web does not require manual labor. In dangerous zones, it is impossible to get close, unlike in normal field surveys. Therefore, this UAV-based system is ideally suited for disaster areas such as volcanos, landslides, and floods.

A sensor web is a group or system of sensors, which connect by using a communication infrastructure to exchange information as a sensor network. In this study, the proposed system considers using a sensor network application. It is a new approach to implement a real-time sensor network with high-density sensor nodes in order to acquire environmental information [5]. The initiative is to develop a GIS and sensor integration system as an infrastructure called sensor service grid (SSG) in order to realize easy deployment and an inexpensive installation and operation of field-server networks. Under this initiative, field-side agent boxes based on sensor observation service (SOS), called SOS stations, have been developed. The use of SOS stations with facilities of the sensor service grid (SSG), under sensor Asia initiative, can simplify these tasks for general users. For this research, UAV has been designed to be one of the sensor nodes of an SOS station.

\section{System Configuration}

3.1. System Architecture. In this study, a digital camera, a gyroscope compass, a laser range meter, and a GPS (global 


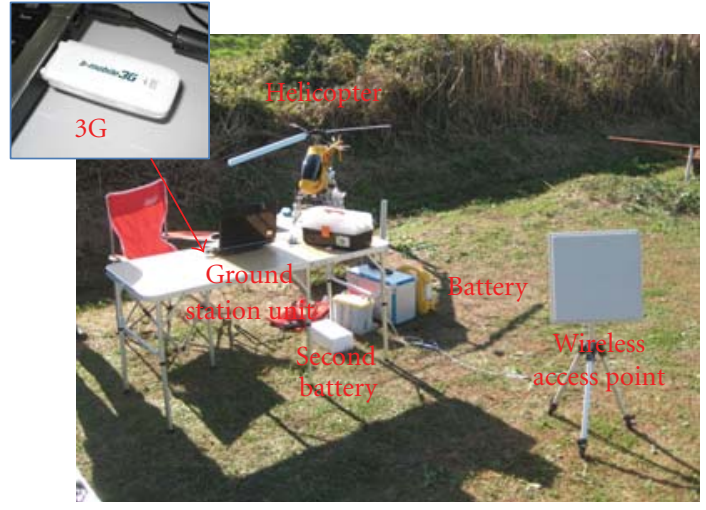

FIgURE 5: S Experiment setup on the ground.

positioning system) are used for the system development. These sensors are mounted on a UAV and controlled by a mini-PC. The acquired information is sent to the ground control station and it is processed and open to the public in real time. Figure 2 shows the system architecture of the UAVbased sensor web monitoring system.

The operator uses RF (radio frequency) remote control to control the UAV. Operation range is up to $300 \mathrm{~m}$ with a height of $60 \mathrm{~m}$. In addition, up to 2 persons can operate the UAV in case of long-range operation, which maybe out of sight range of one operator. Furthermore, the UAV system with SOS extension software can capture images from field areas as well as collect data from sensors and A/D (Analog to Digital) board for connecting sensors. A web server needs to be installed in the system to allow remote control from the ground control station. The communication link is a wireless LAN IEEE $802.11 \mathrm{~g}$. The ground control station is an SOS station with a mobile feature which handles feeding measurement data from the UAV and synchronizing data between SOS and SSG [6]. A wireless connection is used to communicate between the UAV, the ground base station, and the SSG, which enables users to connect to the web application in the ground control station in order to see real-time data. Data feeds from the UAV are automatically synchronized to the SSG. The sensor network is the network of sensor stations, which has SSG as the center of the network. All SOS synchronize both configuration and sensor data to the SSG. A message queue system is used as the media for data synchronization between SOS and SSG, and it gains access to local stations which may be blocked by a firewall or are in private networks. Users can access their stations by both seeing monitored data and controlling the station from the Internet. The UAV system is one of the sensor nodes in the SOS station and SOS will be registered in the sensor network.

A UAV system is developed using Java technology for the core system and $\mathrm{C}++$ language for the device connecting parts. PostgreSQL is chosen as the database because it is a light-weight database, scalable; and moreover, it supports many platforms. Hibernate and Spring frameworks, which are prominent in database management and transaction control, are used as data access layers in this system. The system works as a multithread where all feeders have their own thread as each device has different data rates, such as 1 record per second for GPS, while a gyrocompass is 180 records per second. This technique allows the system to feed data at the maximum speed of each device but it does not need to wait for other devices. Conversely, it generates synchronized data, thus it needs an additional process to synchronize all data on a time basis.

3.2. Sensors. The key elements of the system design are low cost, light weight, ease of use, and mobility. This is because small, commercially available, and relatively inexpensive sensors are used. The digital camera is a Canon PowerShot SX100 IS which features a 10 times optical zoom lens with optical Image Stabilizer, 8.0 Megapixel image sensor (1/2.5"), and DIGIC III processor and runs on two AA alkaline batteries. This model is supported by the Canon PowerShot RemoteCapture Software Development Kit and the camera can be controlled by a computer. The weight is $351 \mathrm{~g}$ and images are captured via the USB interface. The gyroscope compass is MEMS-type IMU (Inertial Measurement Unit) which is produced by InterSense InertiaCube3. It is a precision orientation reference system which uses inertial tracking from the integration of nine sensing elements with advanced Kalman filtering algorithms. It is one of the smallest inertial measurement units and it weighs $155 \mathrm{~g}$. It connects to the RS232 serial interface and runs from a $6 \mathrm{~V}$ power source. The laser range meter used is the TruPulse 200 Rangefinder model and it measures distances and heights. It weighs $378 \mathrm{~g}$ and comes standard with a serial port, offers inscope data display with $7 \mathrm{x}$ power optics, and runs on two AA batteries or a single CRV-3 battery. The GPS receiver to be used is a u-blox AEK-4H GPS device which is a small and lightweight unit with active GPS antenna and uses a builtin USB interface for both power supply and high-speed data transfer, thus eliminating the need for an external power supply. It weighs $255 \mathrm{~g}$. The temperature sensor chosen is National Instruments LM60, which has a linear response in Celsius. The sensor has been mounted on a 10-bit analogto-digital converter card, which has a serial output interface and weighs $103 \mathrm{~g}$. The microclient box is a mini PC with a small form factor and a fan-less design, an x86 CPU of $500 \mathrm{MHz}$ with $1 \mathrm{~GB}$ RAM and it can be booted from a CF card. It is equipped with two RS232 serial interfaces, three USB interfaces, and a single ethernet interface. A mini-PC wireless LAN card is placed internally with external antenna. The weight is $300 \mathrm{~g}$.

3.3. Power Connections and Data Lines. The devices need to connect to the microclient box computing system using different interfaces. As shown in Figure 3, two devices are connected through RS232 serial interface and three devices through USB ports. The figure below sums up the power lines and data connections for all devices in the UAV FS setup.

The ideal power supply for remote-controlled air vehicles is Lithium-Polymer (Li-Po) batteries, which come in cells of $3.7 \mathrm{~V}$ and can be packaged together to get higher voltages. The power supply requirements of each of the components 

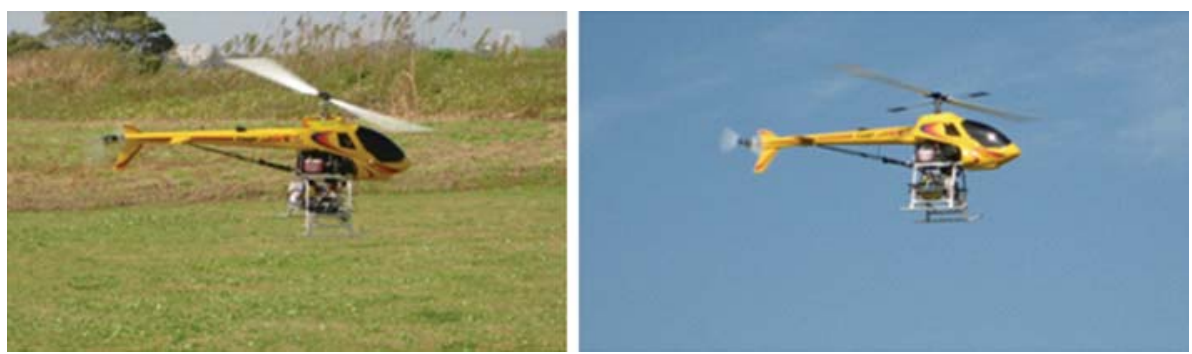

FIGURE 6: UAV with an attachment flying over field area.

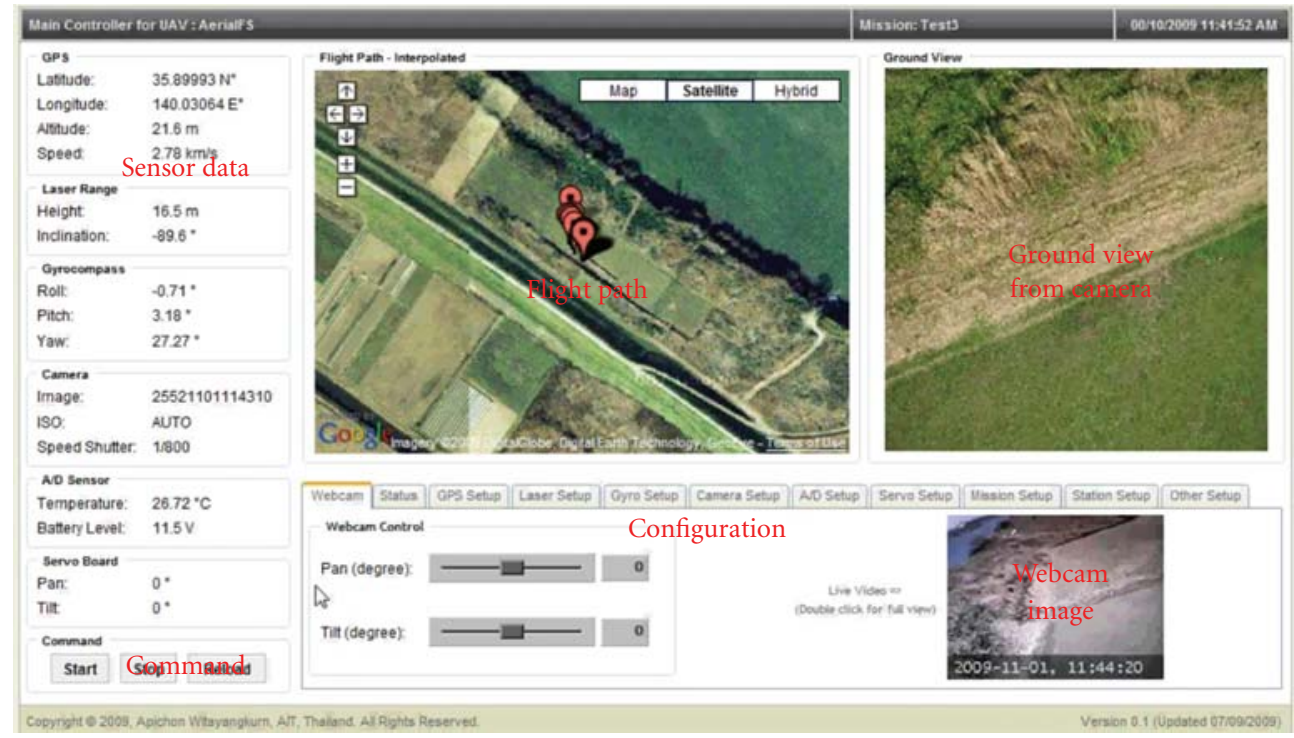

Figure 7: Acquired data.

described above have been considered. The total power consumption of all components is calculated to be 6.3 Watts. A Li-Po battery pack of $11.1 \mathrm{~V}, 1300 \mathrm{mAh}$ is recommended, which can provide continuous power for more than 2 hours. A power supply module was designed and takes $11.1 \mathrm{~V}$ as input and provides output power supplies of $5 \mathrm{~V}$ and $6 \mathrm{~V}$. Two regulators KIC-053 (5 V, $3 \mathrm{~A})$ and 7806 (6 V, $1 \mathrm{~A})$ are the main components. A $1 \mathrm{~A}$ fuse has been connected at the input from Li-Po battery for added protection. Since battery will be used in the platform and it cannot carry heavy size of battery, power consumption of each equipment must be minimized [7].

3.4. Settings. As shown in Figure 4, all the sensors are tightly mounted under the UAV to ensure that they have a constant geometric relationship during the measurement. The CCD cameras are calibrated to estimate relative position and orientation. A web camera is also mounted to send images to the ground station in real time so as to monitor the flight course. Moreover, all sensors are synchronized by GPS time and they are mounted to send images to the ground station in real time to monitor the flight course.

\section{Experimental Test}

An experiment was conducted to test the system. The attachment was tested by being hand held and moved around to check the result and reliability in the first stage. Following this, the hardware platform was attached with the UAV and a flying experiment was conducted with full operation. Voyager GSR 260 was selected for the experiment. The test revealed that the UAV could fly without any problems and the system could also run with all sensors working while flying. Moreover, the ground station could view data and send control commands via a web interface through $\mathrm{Wi}$ Fi. The mobile SOS station at the ground station was able to feed data from the UAV system and could synchronize data to the SSG server in a remote location using a mobile $3 \mathrm{G}$ Internet connection within almost real-time conditions. Figure 5 shows the experiment setup including all related devices for carrying out the experiment. Two batteries were prepared to supply a wireless access point and a laptop. A laptop with Core2Duo $2.2 \mathrm{GHz} 4 \mathrm{~GB}$ Ram was used as a ground station unit (SOS Station) which was connected with an access point to join the same network to the UAV system. To enable Internet access, a b-mobile $3 \mathrm{G}$ modem was used 


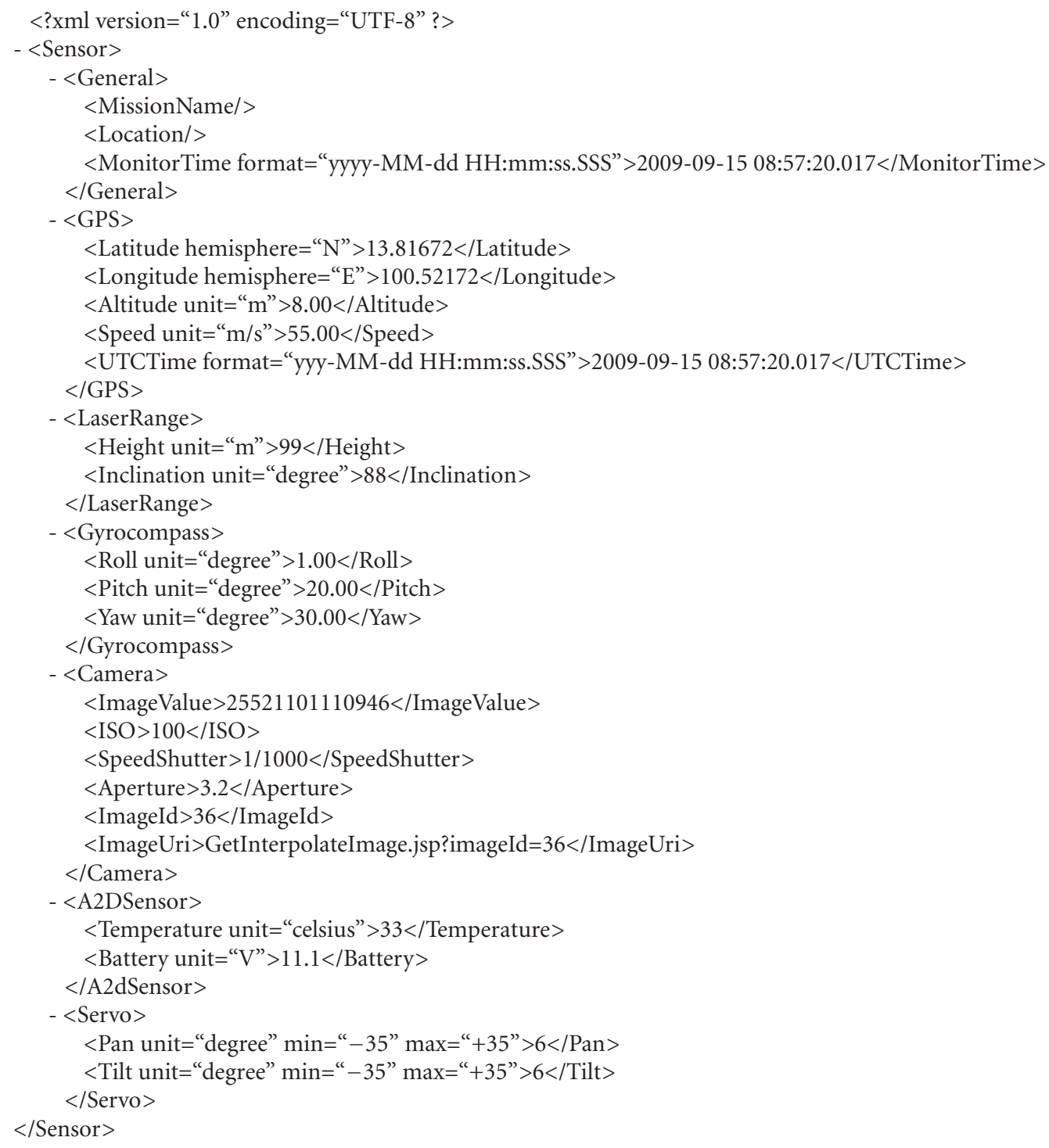

Algorithm 1: XML data format.

via a USB interface. Several flight tests were carried out by turning on the system and starting operation. Figure 6 shows the UAV with an attachment flying over the field area.

\section{Acquired Data}

All sensor connectivity and data acquisition was observed through the Internet. Each feeder for receiving data from the sensors was made. The digital camera and gyroscope compass required a special method called "Java Native Interface (JNI)" to connect devices from Java. For the other sensors, data could be obtained directly from the com port with specific settings. Finally, a core system was developed using Java technology. Hibernate and Spring frameworks are used to control the data access layer. JSP, Servlet, and JSTL are used for the presentation layer. To integrate with a world map location, Google Map is used to show the map and associated pin-point location with latitude and longitude from GPS data. AJAX technology is also used to enhance the system to achieve a more real-time system. Figure 7 shows the main page of the UAV system with the image and the location of the camera, attitude from gyroscope, altitude from laser range meter, and the temperature. Also, other data from ground fixed sensor nodes can be shown through the same site.

Each sensor data must also keep its own location. Furthermore, a data feeder for feeding data from the UAV system is developed. The UAV system provides live interpolated data via an XML data format as shown in Algorithm 1 and can feed via HTTP. Data includes both sensor data and image data. By doing this, users can observe as well as maintain their 
data via a central server. Internet connection is required for data synchronization; however, it does not have to be a fixed public IP but can be any type of Internet connection such as ADSL, Satellite, and GPRS since Message Queue is taken into account under the development of SSG.

\section{Conclusions}

A UAV-based sensor web monitoring system is developed and all the measurement tools are mounted on the UAV to acquire detailed information from a low altitude. The main objective of this study is to design and develop a UAV system as a node of field sensor network which is based on SOS Station and SSG. The development starts from selecting suitable hardware and sensors by using size, weight, and power consumption as the main criteria because the test-bed UAV can have only $3-4 \mathrm{~kg}$ payloads, thus battery pack supplies electricity to all devices. The monitoring is carried out from the sky, but the spatial and temporal resolutions are freely selected near the ground. The data can be easily acquired collectively with safety and mobility by the utilization of a Sensor Web.

A Sensor Web is a type of sensor network which is especially well suited for disaster and environmental monitoring. Sensor nodes are spatially distributed and wirelessly communicate with each other. In this study, a UAV-based system is considered as a mobile sensor node and a combination of UAV-based monitoring with a ubiquitous sensor network is proposed. The system is a web-based application developed by Java Programming and uses PostgreSQL as the database server. Data feeders for each sensor are implemented to connect sensor and feed data. It works as a thread that allows the data feeder to run at the same time. Data is fed from the sensor into the database as well as to the web interface of UAV system.

The UAV system acts as a node of the sensor network and can send its data to the central server and publish for other people to view on the Internet in real time. Finally, system could feed data at one record per second for all sensors except digital camera images that feeds every ten seconds. Mobile SOS station that runs on the ground station unit can obtain interpolated data from the UAV system as well as synchronize both data and image and sensor to central server which is located in another location by using mobile $3 \mathrm{G}$ service within a few minutes, depending on the $3 G$ Internet speed. The system feeds data from all sensors on the UAV and sends data to the central server in almost real time, which is suitable for applications and specialists such as surveillance, rescue, and conducting surveys.

The existing UAV system which is designed to work as a standalone system; thus the advantage of this UAV system can be interoperable with other ground monitoring stations and provide data in real time. As future potential applications, additional sensors can be added to monitor environment such as humidity and $\mathrm{CO}_{2}$ sensor. Also, this system can be improved by increasing performance of hardware such as digital camera for more advanced mapping purpose.

\section{References}

[1] A. Ollero, J. Alcázar, F. Cuesta, and F. López-Pichaco, "Helicopter teleoperation for aerial monitoring in the comets multiuav system," in Proceedings of the 3rd IARP International Workshop on Service, Assistive and Personal, 2003.

[2] K. Honda, A. Shrestha, R. Chinnachodteeranun, A. Witayangkurn, H. N. Duy, and H. Shimamura, "Field sensor network for environmental monitoring-sensor asia initiative and applications," in Proceedings of 3rd International Symposium on Geoinformatics for Spatial Infrastructure Development in Earth and Allied Science (GISIDEAS '08), Hanoi, Vietnam, 2008.

[3] M. Nagai, T. Chen, R. Shibasaki, H. Kumagai, and A. Ahmed, "UAV-borne 3-D mapping system by multi-sensor integration," IEEE Transactions on Geoscience and Remote Sensing, vol. 47, no. 3, pp. 701-708, 2009.

[4] H. Fukui, P. Limlahapun, and T. Kameoka, "Real time monitoring for Imja Glacial Lake in Himalaya-global warming front monitoring system," in Proceedings of the International Conference on Society of Instrument and Control Engineers (SICE '08), pp. 2578-2581, The University Electro-Communications, Tokyo, Japan, 2008.

[5] T. Fukatsu and M. Hirafuji, "Field monitoring using sensornodes with a web server," Journal of Robotics and Mechatronics, vol. 17, pp. 164-172, 2005.

[6] K. Honda, A. Shrestha, A. Witayangkurn, R. Chinnachodteeranun, and H. Shimamura, "Fieldservers and sensor service grid as real-time monitoring infrastructure for ubiquitous sensor networks," Sensors, vol. 9, no. 4, pp. 2363-2370, 2009.

[7] G. Cai, L. Feng, B. M. Chen, and T. H. Lee, "Systematic design methodology and construction of UAV helicopters," Mechatronics, vol. 18, no. 10, pp. 545-558, 2008. 

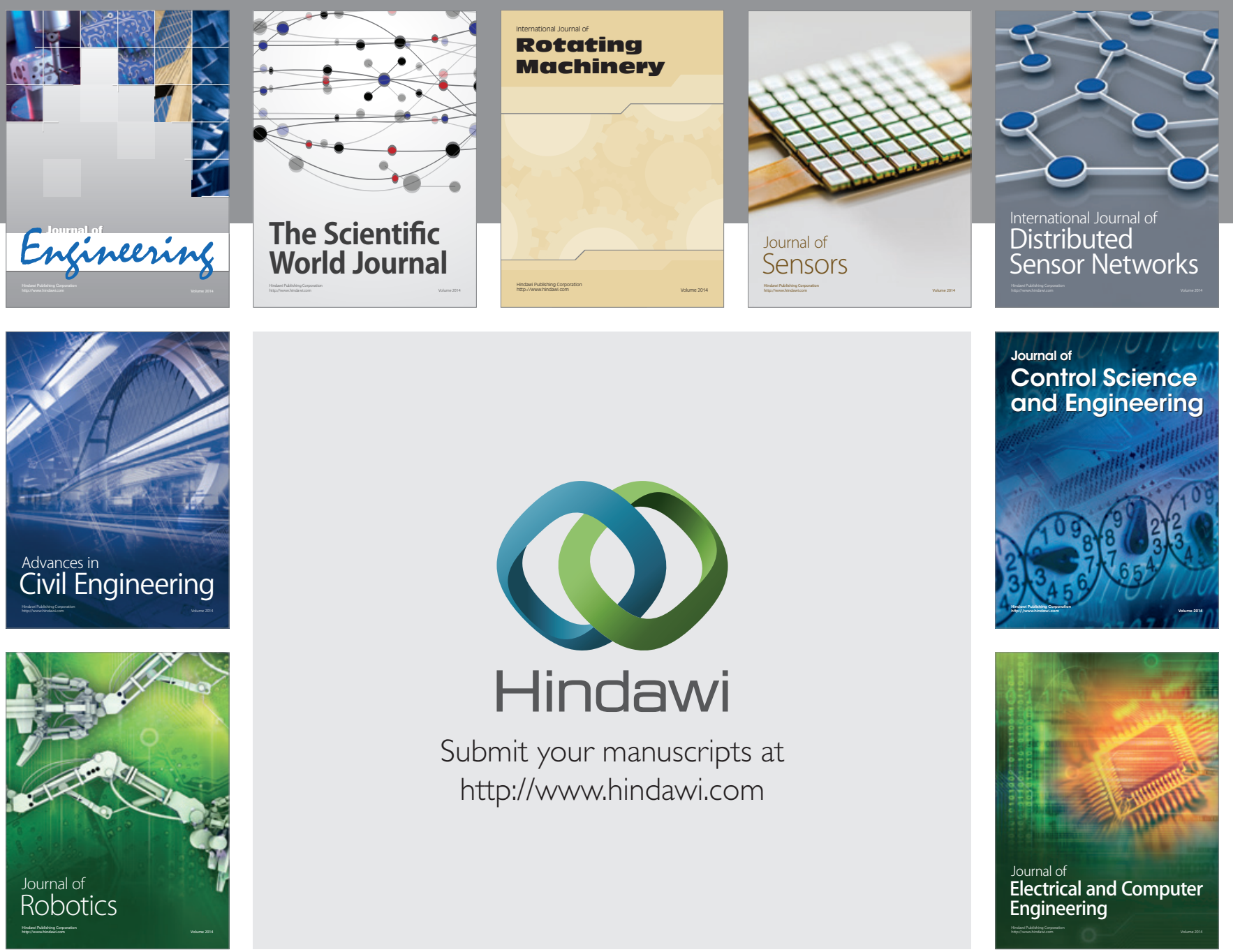

Submit your manuscripts at

http://www.hindawi.com
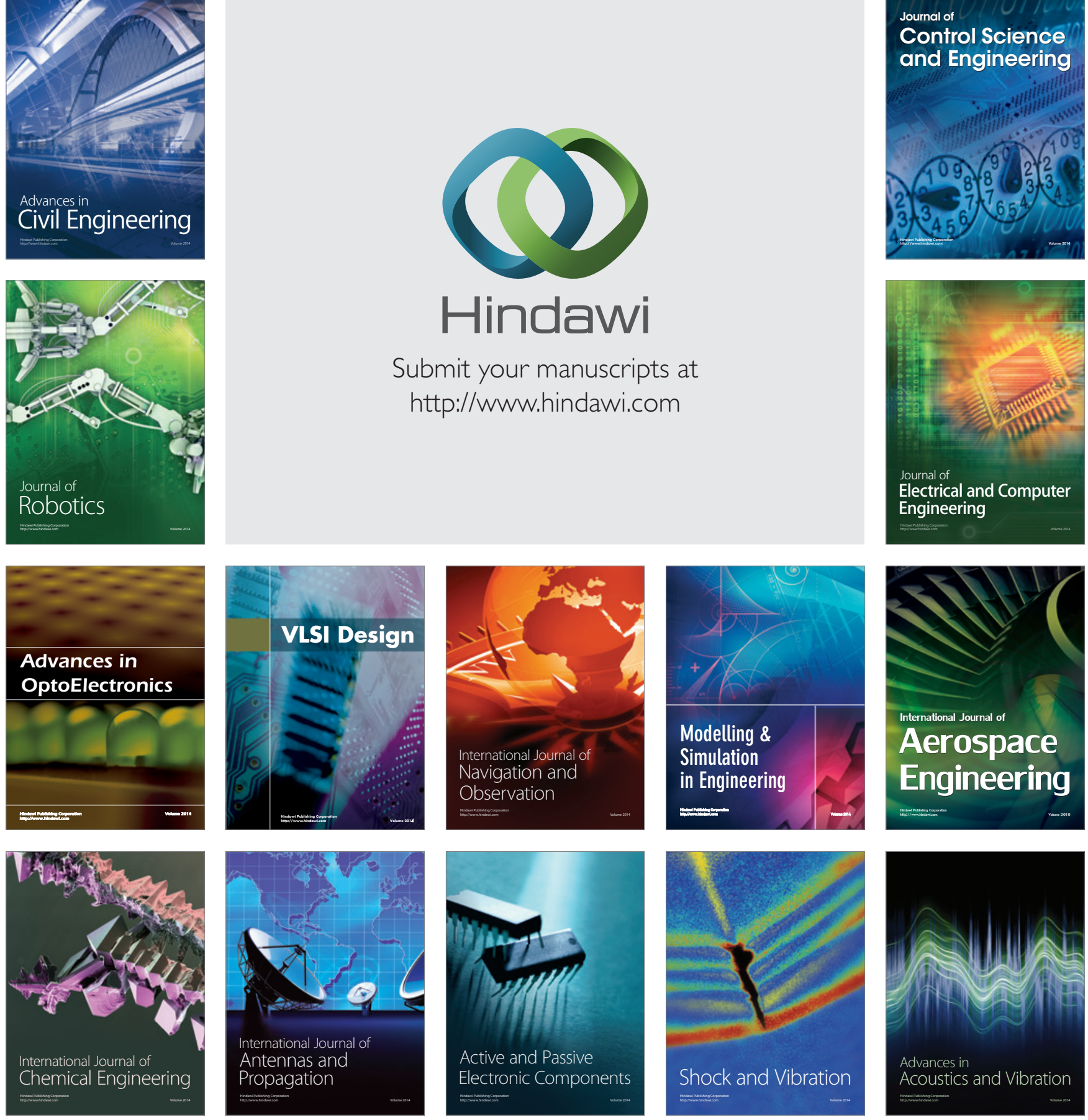